\title{
Building the resource base of research scientists and technologists
}

Countries with knowledge-based economies have witnessed unprecedented material development mainly due to the creation and application of knowledge resulting from research in science and technology. Rapid industrialization, transportation and urbanization followed these developments. This has been accompanied by very significant changes in the life styles of the people, the society as well as the culture of these countries. However, these changes have also led to some very serious problems such as environmental degradation and the rapid depletion of natural resources, among others. The solutions to these problems have also to be sought through research in science and technology. It can be argued that one option to be adopted by developing countries to avoid these problems is through development and or transfer of appropriate knowledge and its assimilation. However, effective development and transfer of knowledge can only be achieved with adequate capital both human and financial, which developing countries lack.

To face these challenges it is therefore essential that countries like Sri Lanka develop self reliance in scientific and technological capabilities by progressively increasing the resource base to a critical mass of well trained scientists and technologists in identified priority areas. The responsibility of human resource development in the areas of science and technology lies basically with the universities, other relevant tertiary level education providers and the research institutes of the country. The faculties of science of the universities produce close upon a thousand graduates annually with three year bachelor's degrees in science. They also award four year bachelor's degrees in certain areas of science. It is necessary for these universities to offer a large number of post graduate programmes at both the Master's and Doctoral levels where research plays the major role. Graduates with such degrees are better equipped for careers in science and technology particularly where research plays a key role. The technical colleges train the technologists needed by the country while it is the research institutes that offer opportunities to young scientists to obtain the much needed experience and expertise.

The institutions that train the human resources necessary for the successful enterprise of science and technology need to be funded adequately without the institutions having to generate funds. Activities for the generation of funds distract the researchers from innovative research which is their main function. Such institutions should be equipped with the necessary infrastructure including state-of-the-art research/teaching equipment. Access to the latest information is another important facility that must be made available to both the staff and students. For the universities and science and technology institutions to generate high quality research and train high calibre research scientists they must be strengthened and modernized. Increasing financial and administrative autonomy and flexibility of science and technology institutions would undoubtedly yield increased quality outputs. In this context, it may also be necessary to amend the relevant acts and regulations.

Developing the necessary manpower for the country is a less difficult task compared to the challenge to retain productive research scientists in the country. The past experience has been one of massive Brain Drain both in the case of scientists and technologists. There are many ways in which this loss can be minimized or prevented. Providing incentives for quality research and innovations would certainly help to retain high calibre recognized scientists, engineers and technologists and to attract Sri Lankan expatriates engaged in these fields to return to the mother country. The incentives may take many forms such as financial rewards for high quality research publications and innovations with potential industrial applications, funding and facilitating patents and funding of doctoral level training.

Improving the working and living conditions of the scientists and technologists, is another important aspect 
that has to be given much consideration to retain them in the country. Enhancement of monthly emoluments to a level comparable with at least the regional levels, provision of health insurance schemes, housing and transportation facilities, and due consideration as a privileged citizen are all worth pursuing. Poor working conditions would certainly result in their looking for better working conditions overseas. To remedy this situation it is necessary where appropriate to establish new centres with advanced international level research facilities in high priority areas. The infrastructure and facilities of the existing research institutions and universities needs to be improved and will go a long way to both retain the researchers and even to attract Sri Lankan scientists working overseas. All research centres and universities must develop links with both local and foreign laboratories, which would help towards exchange of knowledge and researchers. Promotion and establishment of strong partnerships among universities, research institutions and industries for the generation of knowledge and innovation through research is also a very attractive situation for productive researchers. This would provide the researchers additional avenues to generate new knowledge and opportunities to develop new products jointly.

Far reaching success in science and technology research and innovation is the result of commitment by individual researchers or research teams that recognize important problems, think of inspired new solutions and use available resources in the best possible manner to pursue and refine solutions to their ultimate forms. Such researchers are an asset to the nation.

Nalini Ratnasiri 